REFERENCES:

[1] Mortada A Abozaid et al. hydoxychloroquine retinopathy in a ohort of patients from upper Egypt.Journal of Egyptioan Ophthalmological Society 2017:110:110-113

[2] Roy AN,Samala V,Kumar YA,Fatima SS.Assessing the risk of retinopathy in Indian patients using hydroxychloroquine for rheumatic and musculoskeletal Diseases:A Retrospective Observational Study.Indian J Rheumatol 2020.

Disclosure of Interests: None declared

DOI: 10.1136/annrheumdis-2021-eular.2922

\section{POS1398 RHEUMATOLOGICAL PATIENTS PERFORMING BLOOD SELF-SAMPLING FOR DMARD THERAPY SAFETY: A PROOF OF CONCEPT STUDY COMPARING WITH VENOUS BLOOD SAMPLES}

S. A. Just ${ }^{1,2}$, P. Toftegaard ${ }^{1}$, U. Jakobsen ${ }^{3}$, T. R. Larsen ${ }^{2,3} .{ }^{1}$ Svendborg Hospital Odense University Hospital, Section of Rheumatology, Department of Medicine, Svendborg, Denmark; ${ }^{2}$ University of Southern Denmark, Department of Clinical Research, Odense, Denmark; ${ }^{3}$ Svendborg Hospital - Odense University Hospital, Department of Biochemistry, Svendborg, Denmark

Background: Regular blood sampling is a requirement for rheumatological patients receiving csDMARD, bDMARD or tsDMARD therapies (1). The frequent blood sampling affects the patient's life as they use a substantial amount of time at hospitals or by the general practitioner. Often visits are time-consuming with transport, waiting time, and for some patient's costly long travels. Giving patients the option of taking the blood samples themself in their own home, as part of a patient-centred monitoring approach, could provide the patient much higher degree of independence. Further, it may increase the quality of life, cause higher compliance with taking the control samples and possibly reduce health care costs. Objectives: 1. To investigate if rheumatological patients can take capillary blood samples and describe patient-reported outcomes (PRO) about the procedure. 2. Compare the venous and capillary samples' results. 3 . Test if the laboratory automated analysis equipment can handle the small capillary samples.

Methods: 21 rheumatological patients, underwent capillary and venous blood sampling at up to 4 occasions (1-2 months between). Instructions were available on a pictogram. PRO data were assessed by questionnaires. The patient performed blood extraction to the capillary samples from a finger after using a device making a small incision ( $2 \mathrm{~mm}$ depth and $3 \mathrm{~mm}$ width). Two capillary tubes (one Microtainer K2-EDTA and one Microtainer lithium heparin with gel) were filled with a total volume of approximately $1.0 \mathrm{~mL}$ blood. A phlebotomist took the venous sample. Blood samples were analyzed for alanine aminotransferase (ALAT), albumin, alkaline phosphatase (ALP), calcium, C-reactive protein (CRP), creatinine, potassium, lactate dehydrogenase (LDH), urate, hemolysis index, erythrocyte corpuscular volume (MCV), haemoglobin, leukocytes, differential count and platelets.

Results: A total of 53 paired capillary (C) and venous (V) samples were taken. The average perceived pain of the procedure of $C$ sampling was VAS: 10.3 (SD:14.4) (0-100) versus V sampling VAS: 8.5 (SD:11.7). 90\% of patients would accept it as a future form of blood sampling.

Differences in blood samples (C versus V) were: CRP $(-3.4 \%)$; Hemoglobin $(-1.4 \%)$; Creatinine $(-4.4 \%)$, ALAT $(-2.9 \%)$, neutrophils $(1.43 \%)$, platelets $(-16.9 \%)$. The index of hemolysis was on average $128.9 \mathrm{mg} / \mathrm{dL}$ (SD: 203) in C versus $6.7 \mathrm{mg} /$ $\mathrm{dL}$ (SD: 4.6) in V. Results was evaluated by a rheumatologist, and $92.5 \%$ of capillary samples could be used to evaluate the safety of DMARD treatment based on the most critical samples for this: ALAT, creatinine, neutrophils and platelets (1). The $7.5 \%$ not accepted were all due to aggregated platelets leading to low platelet count. There was hemolysis in $18 \%$ of the samples, but the analysis results could be used despite this. Conclusion: In the majority of rheumatological patients, capillary self-sampling is well tolerated.

We show that it is possible to extract the needed results from the capillary samples to evaluate DMARD treatment safety, despite higher hemolysis index. Using capillary samples taken at home could be a central instrument in future rheumatological patient-centred monitoring.

REFERENCES:

[1] Rigby WFC et al. Review of Routine Laboratory Monitoring for Patients with Rheumatoid Arthritis Receiving Biologic or Nonbiologic DMARDs. Int $J$ Rheumatol. 2017

Disclosure of Interests: None declared

DOI: 10.1136/annrheumdis-2021-eular.2927

\section{POS1399 \\ ALGORITHM FOR DIFFERENTIAL DIAGNOSIS OF ARTHROPATHIES USING OSTEOSCINTIGRAPHY}

E. Prokhorova ${ }^{1}$,E. Zhilyaev ${ }^{2}$, G. Zhilyaev ${ }^{3}{ }^{1}$ Main Hospital of Police, Nuclear Imaging, Moscow, Russian Federation; ${ }^{2} J S C$ European Medical Center, Medical Administration, Moscow, Russian Federation; ${ }^{3}$ Moscow State University of Medicine and Dentistry named after A.I. Evdokimov, Student, Moscow, Russian Federation
Background: Joint bone scintigraphy is a promising method for diagnosing of inflammatory joint diseases. However, its scintigraphic semiotics remains to be developed.

Objectives: To develop an algorithm for the differential diagnosis of the most common arthropathies based on quantitative osteoscintigraphy.

Methods: This single-center study included inpatients with clinical diagnoses of rheumatoid arthritis (RA), axial spondyloarthritis (including ankylosing spondylitis - aSPA), peripheral spondyloarthritis (including reactive arthritis - $\mathrm{pSpA}$ ), psoriatic arthritis (PsA), and osteoarthritis (OA) established by a rheumatologist and meeting the relevant criteria, Three hours after the injection of pyrophosphate, labeled with Tc-99m, scintigraphy of the skeletal bones was carried out according to the "whole body" program. The joint / bone accumulation ratio (AR) was calculated as the ratios of counts in relevant areas. All AR were recalculated into T-score for each joint based on data from control group. The CHAID algorithm for classification trees constructing was used. The significance of the division in the nodes was estimated with Bonferroni adjustment.

Results: 266 patients were included in the study aged $46.6 \pm 14.3$ years, men $134(50.4 \%)$. aSPA was diagnosed in 40 patients, pSpA in 87, RA in 45, OA in 68 , PsA in 26. 2279 joints were analyzed. A classification tree for differential diagnosis of arthropathies has been built (Pict.). Key indicators for identifying subgroups in the algorithm: AR in the wrist, knee and hip joints.

At the first step of the classification tree, the sum of the T-scores of the wrist joints is determined. If this amount exceeds 7.76 (node 2), the most likely diagnosis is RA ( $58.5 \%$ of patients in the subgroup of this node). Patients with the sum of the T-scores in the wrist joints less than 7.76 (node 1), in turn, were divided depending on the sum of the T-scores of hip joints. Those with this value less than 3.25 (node 3), mainly suffered from OA (48.7\%), less often in this subgroup were diagnosed pSpA $(16.7 \%)$ and PsA (14.1\%). Patients in whom the sum of the T-score in the hips was more than 3.245 (node 4) were further subdivided depending on the sum of the T-scores in the shoulder joints. If it was more than 4.21 (node 8), then pSpA was most likely (58.7\%). If the sum of the T-scores of the shoulder joints was in the range from 2.05 to 4.21 (node 7 ), then these are mainly patients with spondyloarthritides $(56.2 \%$ with aSPA and $31.2 \%$ with pSpA) and a small number of patients (10\%) with PsA. In the subgroup with the sum of T-scores of the shoulder joints $<2.05$ (node 6), patients with PSA (40\%) prevailed, with a significant proportion of persons with OA (27.5\%). Thus, out of the final branches formed by the tree (node 2, 3, nodes 6-8), four correspond to certain diseases (node 2 - PA, node 3 - OA, node 7 - aSPA, node 8 - pSpA). Node 6 includes patients with various diseases with a predominance of PsA. The level of significance of differences between the formed groups at all branch points of the tree is $p=0.001$ or less (Bonferroni adjusted). In the training sample, $51.5 \%$ of observations are correctly classified. According to cross-validation data, the expected rate of correct classifications in real application of the algorithm is $38.0 \%$.

Conclusion: An algorithm for the differential diagnosis of the most common inflammatory diseases of the joints has been developed, which makes it possible to use the data of quantitative osteoscintigraphy in the process of diagnosing arthritis.

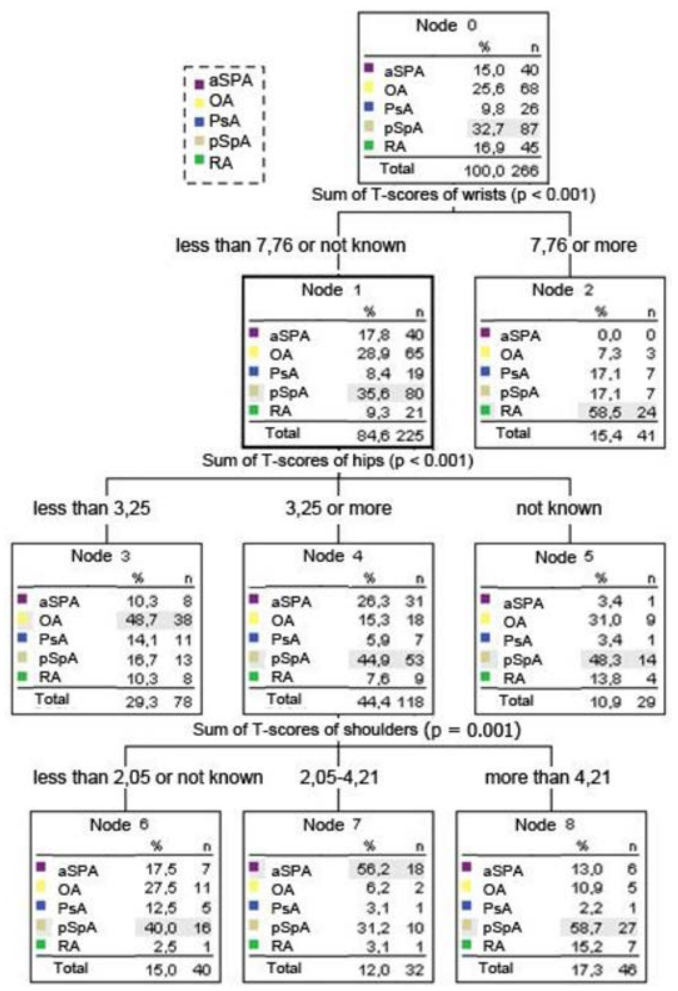


Acknowledgements: MD. PhD Constantin V. Kushnir, Main hospital of police.

Disclosure of Interests: None declared

DOI: 10.1136/annrheumdis-2021-eular.2931

\section{POS1400 A SYSTEMATIC REVIEW OF INTERNATIONAL GUIDELINES REGARDING THE ROLE OF RADIOGRAPHY IN THE DIAGNOSIS OF OSTEOARTHRITIS}

C. Henry-Blake ${ }^{1}$, K. Treadwell ${ }^{1}$, S. Parmar ${ }^{1}$, J. Higgs ${ }^{1}$, M. Marshall ${ }^{1}$, J. Edwards', G. Peat ${ }^{1}{ }^{1}{ }^{1}$ Keele University, Department of Primary, Community and Social Care, Keele, United Kingdom

Background: A substantial proportion of primary care osteoarthritis (OA) consultations are associated with an X-ray request ${ }^{(1,2)}$. Uncertainty exists regarding the ability of radiography to improve a clinical OA diagnosis, and the over-use of radiography may lead to inappropriate referrals due to severe radiographic features that do not correlate with patients' symptoms. Additionally, there are cost implications of unnecessarily imaging such a prevalent disease. As evidence questions the utility of routine radiography in $\mathrm{OA}$, the extent to which radiography is supported by international guidelines is unknown.

Objectives: To undertake a systematic review and narrative synthesis of UK and international guideline recommendations on the role of radiography in the diagnosis of $\mathrm{OA}$

Methods: A systematic search of eleven electronic databases (including EMBASE, MEDLINE CINAHL, Epistemonikos and Guideline Central) and the websites of nine professional organisations (including NICE, Royal College of Radiologists (RCR), EULAR, and the American College of Radiology $(A C R)$ ) identified the most recent evidence-based guidelines produced by professional organisations on the role of imaging in OA. Guidelines not addressing the role of radiography in the diagnosis of OA were excluded, as were non-English and spinal OA guidelines. Each title was screened by one reviewer whilst each abstract and full text underwent dual screening. A single reviewer, using a standard proforma, undertook data extraction. Each guideline was independently appraised by two reviewers using the AGREE II tool. A narrative synthesis of the nature and consistency of OA radiographic recommendations was performed.

Results: 18 evidence-based OA guidelines published between 1998-2019 were included. These guidelines considered OA at any joint $(n=8)$, or at the knee $(n=3)$, hip $(n=2)$, hand $(n=2)$, wrist $(n=1)$, foot $(n=1)$, and ankle $(n=1)$. Seven guidelines were produced by European organisations; four guidelines were produced by EULAR. Guidelines were targeted at general practitioners $(n=11)$, radiologists $(n=7)$, rheumatologist $(n=4)$ and orthopaedic surgeons $(n=3)$. Using the AGREE II tool, the identified guidelines scored highly on rigour of development (mean score 69\%) but poorly on applicability (32\%). All 18 guidelines recommended X-rays as the first-line modality, where imaging was indicated. A clinical diagnosis of OA without radiographic confirmation was recommended by all eleven guidelines produced by organisations represented general practitioners, with seven guidelines justifying this due to a poor correlation between radiographic features and clinical symptoms. Only three guidelines explicitly discouraged the routine use of radiography for the diagnosis of $O A$ and only two guidelines reassured practitioners of a low probability of missing serious pathology when not routinely requesting radiographs. Guidelines produced by organisations representing radiologists were more supportive of radiography. The ACR recommended radiographic confirmation in patients suspected to have OA at the hand, wrist, hip, knee, ankle, and foot. Conversely, the RCR recommended radiographic confirmation in patients suspected to have $\mathrm{OA}$ at the hand, feet, and hip, but not the knee.

Conclusion: Differences in guideline recommendations on the utility of radiography in OA appear related to country/region, professional organisation, and joint. The use and utility of radiography in OA may need to be reviewed in light of a shift towards remote consultations, a change that has been accelerated by COVID-19 in many countries.

REFERENCES:

[1] Yu D, Jordan K, Bedson J, Englund M, Blyth F, Turkiewicz A et al. Population trends in the incidence and initial management of osteoarthritis: age-period-cohort analysis of the Clinical Practice Research Datalink, 1992-2013. Rheumatology. 2017;56(11):1902-1917.

[2] Brand C, Harrison C, Tropea J, Hinman R, Britt H, Bennell K. Management of Osteoarthritis in General Practice in Australia. Arthritis Care \& Research. 2014;66(4):551-558

Acknowledgements: JJE is funded by an Academic Clinical Lectureship from the National Institute for Health Research (NIHR) for this research project (CL2016-10-003). The views expressed in this publication are those of the author(s) and not necessarily those of the NIHR, NHS or the UK Department of Health and Social Care.

Disclosure of Interests: None declared

DOI: 10.1136/annrheumdis-2021-eular.3117

\section{POS1401 ASSESSMENT OF INTERREADER RELIABILITY IN SCORING PATIENTS WITH HAND OSTEOARTHRITIS AND PSORIATIC ARTHRITIS BY FLUORESCENCE OPTICAL IMAGING}

B. Drude ${ }^{1}, \varnothing$. Maugesten ${ }^{2}$, S. G. Werner ${ }^{3}$, G. R. Burmester ${ }^{1}$, J. Berger $^{4}$, I. K. Haugen ${ }^{2}$, S. Ohrndorf ${ }^{1}{ }^{1}$ Charité Universitätsmedizin Berlin, Department of Rheumatology and Clinical Immunology, Berlin, Germany; ${ }^{2}$ Diakonhjemmet Hospital, Department of Rheumatology, Oslo, Norway; ${ }^{3} \mathrm{RHIO}$ (Rheumatology, Immunology, Osteology) Duesseldorf, RHIO Research Institute, Duesseldorf, Germany; ${ }^{4}$ Xiralite GmbH, Head Development and Produktion, Berlin, Germany

Background: Fluorescence Optical Imaging (FOI) utilises the fluorophore indocyanine green (ICG) to reflect enhanced microcirculation in hand and finger joints due to inflammation.

Objectives: We wanted to assess the interreader reliability of $\mathrm{FOI}$ enhancement in patients with hand osteoarthritis (OA) and psoriatic arthritis (PsA). Furthermore, predefined typical morphologic patterns were included to determine the ability of FOI to discriminate between both diagnoses.

Methods: An atlas with example images of grade 0-3 in different joint groups and typical morphologic patterns ('streaky signals'[1], 'green/blue nail sign'[2], 'Werner sign'[3,4], and 'Bishop's crozier sign') of PsA and hand OA was created. Two readers scored all joints in both hands ( 30 in total) of 20 cases with hand OA and PsA. The cases were randomly mixed and both readers were blinded to diagnosis. Each joint was rated on a semiquantitative scale from 0 to 3 in five different images (PrimaVista Mode (PVM), phase 1, 2 (first and middle image), and 3) during the FOI sequence according to the scoring method FOIAS (fluorescence optical imaging activity score) $[1,3]$. Interreader reliability on scoring joint enhancement was calculated using linear weighted Cohen's kappa (к). Agreement on diagnosis (hand OA vs. PsA) and different morphologic patterns was assessed by calculating (regular) Cohen's kappa.

Results: Overall agreement on scoring joint enhancement (all phases) was substantial $(\kappa=0.75)$, with greatest consensus in phase 2 first $(\kappa=0.75)$ and lowest agreement in phase $1(\kappa=0.46)$. Reliability varied in different joint groups (wrist, MCP, (P)IP, DIP), with almost perfect overall agreement on PIP joint affection $(\kappa=$ $0.81)$, substantial agreement on wrist $(\kappa=0.69)$ and DIP joint affection $(\kappa=0.63)$ and moderate agreement on MCP joint affection $(\kappa=0.49)$ across all phases. Consensus on morphologic patterns showed overall fair agreement $(\kappa=0.37)$ with a similar kappa value on the ability to discriminate between both diagnoses $(\kappa=0.3)$. Conclusion: Joint enhancement in FOI can be reliably assessed using a predefined scoring method. The ability of FOI to differentiate between hand OA and PsA seems to be limited. Clearer definition and more training might be needed to better agree on morphologic patterns in FOI.

REFERENCES:

[1] Glimm AM, Werner SG, Burmester GR, et al. Ann Rheum Dis. 2016 Mar;75(3):566-570

[2] Wiemann O, Werner SG, Langer HE, et al. J Dtsch Dermatol Ges. 2019 Feb;17(2):138-148

[3] Werner SG, Langer HE, Ohrndorf S, et al. Ann Rheum Dis. 2012 Apr;71(4):504-510

[4] Zeidler H 2019. Fluoreszenzoptische Bildgebung. In: Zeidler H, Michel BA Differenzialdiagnose rheumatischer Erkrankungen 5. Aufl. Springer, Heidelberg, S. 88-89

Disclosure of Interests: Benedict Drude: None declared, Øystein Maugesten: None declared, Stephanie Gabriele Werner: None declared, Gerd Rüdiger Burmester: None declared, Jörn Berger Employee of: Xiralite GmbH, Ida K. Haugen: None declared, Sarah Ohrndorf: None declared DOI: 10.1136/annrheumdis-2021-eular.3145

\section{POS1402 HIGHER PREVALENCE OF SUBCLINICAL ATHEROSCLEROSIS IN PSORIATIC ARTHRITIS PATIENTS}

S. Lugo-Perez ${ }^{1}$, I. J. Colunga-Pedraza², D. Á. Galarza-Delgado ${ }^{2}$, J. R. AzpiriLópez $^{1}$, A. B. Rodriguez-Romero ${ }^{2}$, N. Guajardo-Jauregui ${ }^{2}$, D. E. Flores Alvarado $^{2}$, A. Cárdenas ${ }^{2}$, A. C. Garza Acosta ${ }^{3}$, O. Ilizaliturri Guerra², G. GarcíaArellano ${ }^{2}{ }^{1}$ Hospital Universitario "Dr. José Eleuterio González", Universidad Autónoma de Nuevo León, Cardiology, Monterrey, Mexico; ${ }^{2}$ Hospital Universitario “Dr. José Eleuterio González", Universidad Autónoma de Nuevo León, Rheumatology, Monterrey, Mexico; ${ }^{3}$ Hospital Universitario "Dr. José Eleuterio González", Universidad Autónoma de Nuevo León, Radiology, Monterrey, Mexico

Background: Psoriatic arthritis (PsA) patients have a higher risk of developing a cardiovascular (CV) event than the general population due to an increased prevalence of traditional $\mathrm{CV}$ risk factors and to disease characteristics such as disease duration and activity. The carotid ultrasound (US) is a non-invasive diagnostic tool that can detect the presence of subclinical atherosclerosis which is directly associated with the risk of developing a CV event.

Objectives: The aim of this study is to compare the prevalence of subclinical atherosclerosis detected by carotid US in PsA patients and controls. 